\title{
The efficacy of recovery care with cool veil serum
}

\author{
Yu Yumoto, Takehiko Yukishita, Keiko Lee, Hiroyuki Kobayashi
}

Department of Hospital Administration, Graduate School of Medicine, Juntendo University School of Medicine, Tokyo, Japan; *Corresponding Author: yuyumoto1@gmail.com

Received 28 September 2010; revised 18 October 2010; accepted 22 October 2010

\begin{abstract}
The purpose of this study was to investigate the efficacy of post-exercise treatment with "Cool Veil Serum (CVS)" developed by Madre:X Co. Ltd. (Shibuya, Tokyo) to prevent exercise-induced muscle swelling. Calf circumferences and muscle stiffness of both legs were measured in 10 healthy female. The measurements were made on before 5-minute exercise, after the exercise, and after 10-minute recovery care with CVS and 5-minute rest. The recovery care was applied only on right leg of the subjects. Then, multiple comparisons of calf circumference and muscle stiffness on each leg among pre-exercise, post-exercise, and final measurements were carried out. Exercise caused a significant increase in calf circumference and muscle stiffness. The recovery care with CVS rapidly reduced post-exercise calf circumference to pre-exercise level. Relationship between reducing muscle swelling after exercise and preventing sport injury should be explored in further studies.
\end{abstract}

Keywords: Recovery Care; Cool Veil Serum; Massage after Exercise; Calf Circumference; Muscle Stiffness

\section{INTRODUCTION}

Growing health consciousness in recent years is inspiring many people to lead an active lifestyle. According to the 2008 Sports Life Survey, recreationally active individuals who engage in "moderate intensity exercise twice a week for 30 min" for health promotion and maintenance constituted a record-breaking $45.5 \%$ of the population since the survey first began in 1992. In addition, the number of people participating in physical activity and sports has risen to $56.4 \%$ [1]; indeed more than 50 million Japanese people are currently exercising on a weekly basis.
Meanwhile the 2008 National Health and Nutrition Survey showed that one in two men and one in five women aged 40-74 years are afflicted with pre-metabolic and metabolic syndrome in Japan [2]. As dietary changes and exercise programs become the cornerstones of treatment for metabolic syndrome, the physically active population is anticipated to increase further.

The rising exercise trend reflects a shift towards positive health promotion, however, a commensurate overall increase in sports-related injuries and fatigue-induced pain is expected as well. In general, athletes are more conscious about post-exercise recovery and have easier access to knowledgeable trainers and therapists. Nonetheless among the collegiate athletes who are educated in recovery care more so than ordinary people, only half confess to stretching before and not after workouts [3]. Additionally, the number of certificated athlete trainers by Japan Sports Association is only 1,356 in Japan [4], while more than 30,000 athletic trainers belonging to National Athletic Trainers' Association are available in the United States [5]. Therefore, there have been few non-athletes exercising with certified athletic trainers in Japan. In light of these findings, recreationally active people are at higher risk of foregoing post-workout recovery care. Consequently this very paucity of awareness and knowledge appears to promote sports injuries.

Despite the importance of post-workout recovery strategies as lauded by healthcare professionals and athletic trainers, simple post-exercise body care for ordinary people are currently not available. Accordingly we created "Cool Veil Serum (CVS)" for post-workout recovery care that regular people can routinely use to facilitate cool down and prevent fatigue by massaging into exercised muscles. Our intention is to elevate consciousness about recovery care with a handy product like CVS that provides additional benefits of protecting the skin, restoring vitality, and enhancing the circulation. In the present study, we evaluated the effect of "Cool Veil Serum" application after a workout in preventing exercise-induced muscle swelling. 


\section{MATERIALS \& METHODS}

\subsection{Cool Veil Serum (CVS)}

The Body Hygiene Cool Veil Serum was created by Madre: X Co. Ltd (Shibuya, Tokyo) to deliver a product that at once prevents fatigue, cools working muscles, and protects the skin when applied after physical activity. Ingredients such as BCAA, glutamic acid, alginine, and citric acid have been blended to hasten recovery. Menthol has been added to cool muscles. In addition, this lotion has been enriched with skin emolliating microcollagen and hyaluronic acid and renders the dual benefit of nourishing the skin and improving blood and lymph flow when applied on working muscles. Hydrangeic acid, a compound derived from Hydrangea macrophylla var. thunbergii, has been reported to upregulate adiponectin expression and thereby reduce triglycerides and blood glucose levels [6], potentially halting the genesis of metabolic syndrome.

\subsection{Study Participants}

Ten healthy, regularly active female subjects aged 21-35 participated in the study after providing oral consent. Individuals who are pregnant or possibly pregnant, or unable to perform weight-bearing exercises were excluded from the study.

\subsection{Methods}

We used five steps to study the efficacy of post-exercise recovery care with CVS.

\section{1) Pre-exercise (baseline) measurements}

The present study was conducted at our research institute. Baseline calf circumference and muscle stiffness of both legs were measured before the test exercise. The circumference of the most prominent aspect of the gastrocnemius muscle served as the reference body part. The gastrocnemius circumference was measured by hand and NEUTONE muscle hardness tester (TRY-ALL COPORATION, TDM-NAI, Chiba, Japan) was employed to evaluate muscle stiffness. A semi-permanent ink pen was used to create landmarks on the skin of the gastrocnemius to allow repeated measures to be taken at the same location each time. Final measurements were taken after confirming the reliability of test methods through several practice measurements.

2) Weight-bearing exercise for the lower legs

Each participant performed 150 bilateral heel lifts in a temperature-controlled (23C) room. A trainer supervised the 5-min test exercise to ensure that stress load and pace of all participants were consistent.

\section{3) Post-exercise measurements}

The gastrocnemius circumference and muscle stiff- ness were measured immediately after the heel lift test exercise.

\section{4) Recovery care with CVS}

Following post-exercise measurements, each participant performed a 10-min self-application with CVS only on the right leg. The left leg, served as control, was in rest position during the right-leg recovery care. Recovery care was performed as follows:

a) Apply $10 \mathrm{ml}$ of CVS to right posterior calf and thigh slowly (repeat 20 times for about $7 \mathrm{~min}$ ).

b) Apply $10 \mathrm{ml}$ of CVS to right lateral shin and thigh slowly (repeat 10 times for about $3 \mathrm{~min}$ ).

\section{5) Final measurements}

The final calf circumference and muscle stiffness were measured after the 10-min self-application and 5-min rest.

\subsection{Data Analysis}

Student's t-test was used to determine pre-exercise difference between the legs. As the leg-to-leg comparison model was used to determine the efficacy of test lotion (CVS) in recovery care, our analysis thence proceeded on the assumption that baseline measurements did not reveal striking left-right differences.

Repeated measures analysis of variance (ANOVA) and Turkey's method of multiple comparisons were run to capture the differences among pre-exercise, post-exercise, and final measurements. Statistical significance was set at $\mathrm{P}<0.05$ for all analyses. Data analysis was performed using a statistical software package (SPSS Ver. 11.5).

\section{RESULT}

\subsection{Results of Measurements}

Table 1 summarizes the pre-exercise, post-exercise, and final measurements of the gastrocnemius of the 10 study participants. (Table 1-1: Calf circumference, Table 1-2: Calf stiffness)

\subsection{Left-Right Pre-Exercise Differences}

No significant differences were found in baseline circumference and stiffness between the legs (calf circumference: $p=0.835$, muscle stiffness: $p=0.884$ ). We therefore treated the two sides as symmetrical and conducted further analysis based on these findings.

\subsection{Comparisons among Pre-Exercise, Post-Exercise, and Final Measurements}

\subsubsection{Calf circumference}

Figure 1 depicts the means of pre-exercise, post- 
Table 1-1. Right and left calf circumferences of the subjects on pre-exercise, post-exercise and final measurements $(\mathrm{N}=10)$.

\begin{tabular}{ccccccccc}
\hline & & \multicolumn{3}{c}{ CVS(Right) } & \multicolumn{5}{c}{ Control(Left) } \\
\hline Subjects & Age & Pre-exercise & Post-exercise & Final & Pre-exercise & Post-exercise & Final \\
1 & & & & & & 32.8 & 33.4 & 32.6 \\
2 & 19 & 33.0 & 33.2 & 32.4 & 34.1 & 34.8 & 36.3 & 35.5 \\
3 & 24 & 34.3 & 35.7 & 34.0 & 34.6 & 34.2 \\
4 & 28 & 34.4 & 35.0 & 34.2 & 34.0 & 33.8 & 34.0 \\
5 & 28 & 32.9 & 31.0 & 33.5 & 34.0 & 31.5 & 31.5 \\
6 & 28 & 31.0 & 34.9 & 34.4 & 34.6 & 35.2 & 34.7 \\
7 & 35 & 34.5 & 34.1 & 33.6 & 32.0 & 34.0 & 33.4 \\
8 & 31 & 33.5 & 35.5 & 34.8 & 33.2 & 35.0 & 34.7 \\
9 & 31 & 35.2 & 36.6 & 36.2 & 35.5 & 36.9 & 36.6 \\
10 & 31 & 38.2 & 39.7 & 38.1 & 37.4 & 39.4 & 38.4 \\
Average & 28.6 & 34.2 & 35.0 & 34.2 & 34.0 & 35.0 & 34.6 \\
SD & 4.5 & 1.9 & 2.3 & 2.1 & 1.7 & 2.2 & 2.0 \\
\hline
\end{tabular}

Table 1-2. Right and left calf muscle stiffness of the subjects on pre-exercise, post-exercise and final measurements $(\mathrm{N}=10)$.

\begin{tabular}{|c|c|c|c|c|c|c|c|}
\hline \multirow[b]{2}{*}{ Subjects } & \multirow[b]{2}{*}{ Age } & \multicolumn{3}{|c|}{ CVS(Right) } & \multicolumn{3}{|c|}{ Control(Left) } \\
\hline & & Pre-exercise & Post-exercise & Final & Pre-exercise & Post-exercise & Final \\
\hline 1 & 19 & 33.5 & 34.0 & 34.0 & 33.0 & 35.0 & 34.0 \\
\hline 2 & 24 & 29.0 & 30.5 & 30.0 & 28.0 & 30.0 & 29.5 \\
\hline 3 & 28 & 32.0 & 34.0 & 32.0 & 33.0 & 37.0 & 34.0 \\
\hline 4 & 28 & 30.0 & 32.0 & 30.0 & 31.0 & 34.0 & 32.0 \\
\hline 5 & 28 & 34.0 & 39.0 & 37.0 & 34.0 & 38.5 & 37.5 \\
\hline 6 & 35 & 31.0 & 32.0 & 31.0 & 31.0 & 32.5 & 31.0 \\
\hline 7 & 31 & 28.5 & 29.0 & 28.5 & 28.5 & 30.0 & 29.0 \\
\hline 8 & 31 & 35.0 & 34.0 & 32.0 & 34.0 & 33.0 & 33.0 \\
\hline 9 & 31 & 31.0 & 33.0 & 31.0 & 31.0 & 34.0 & 32.0 \\
\hline 10 & 31 & 28.0 & 34.0 & 33.0 & 30.0 & 34.0 & 34.0 \\
\hline Average & 28.6 & 31.2 & 33.2 & 31.9 & 31.4 & 33.8 & 32.6 \\
\hline SD & 4.5 & 2.4 & 2.7 & 2.4 & 2.1 & 2.7 & 2.5 \\
\hline
\end{tabular}

exercise and final circumference. Significant baseline and post-exercise differences (left and right: $\mathrm{p}<0.001$ ) confirmed that exercise caused a marked increase in calf circumference. The mean circumference of the CVSapplied (right) leg was significantly less compared with the mean of post-exercise circumference $(\mathrm{p}<0.001)$. In contrast, the control (left) leg did not show a striking difference between post-exercise and final circumference $(p=0.090)$. Moreover, the final circumference of the left leg was significantly greater than pre-exercise circumference $(p=0.025)$.

\subsubsection{Muscle Stiffness}

The means of pre-exercise, post-exercise, and final stiffness are evident in Figure 2. Significant lateralized differences between pre- and post-exercise stiffness (left: $p<0.001$, right: $p=0.007)$ confirmed that exercise induced prominent muscle stiffness. A small difference was noted between the means of post-exercise and final stiffness of the CVS-applied (right) leg, albeit statistically insignificant $(\mathrm{p}=0.080)$. In contrast, the control (left) leg showed a significant difference between postexercise and post-immobilization muscle stiffness $(p=$ 0.035). Unlike the right leg, stiffness of the left leg remained significantly greater after immobilization compared with baseline $(\mathrm{p}=0.027)$.

\section{DISCUSSION}

We found that recovery care with CVS rapidly re- 


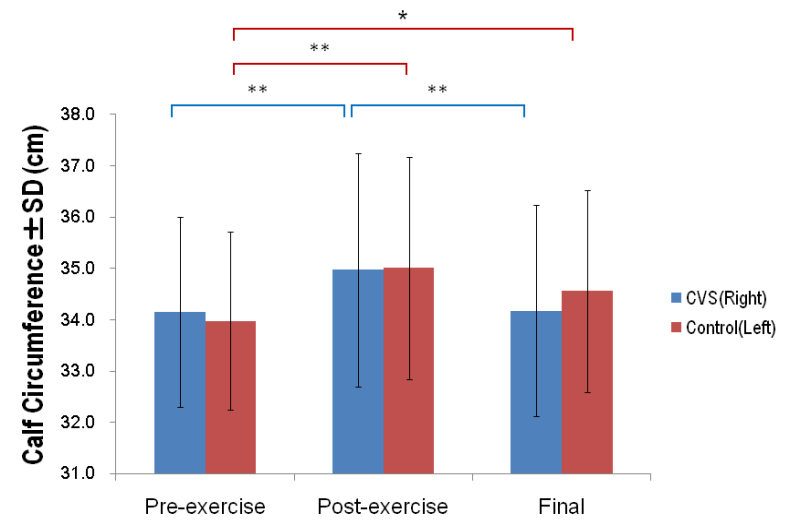

Figure 1. Multiple comparisons among pre-exercise, postexercise and final measurements of right and left calf circumferences $(*: \mathrm{p}<0.05, * *$ : $\mathrm{p}<0.01)$.

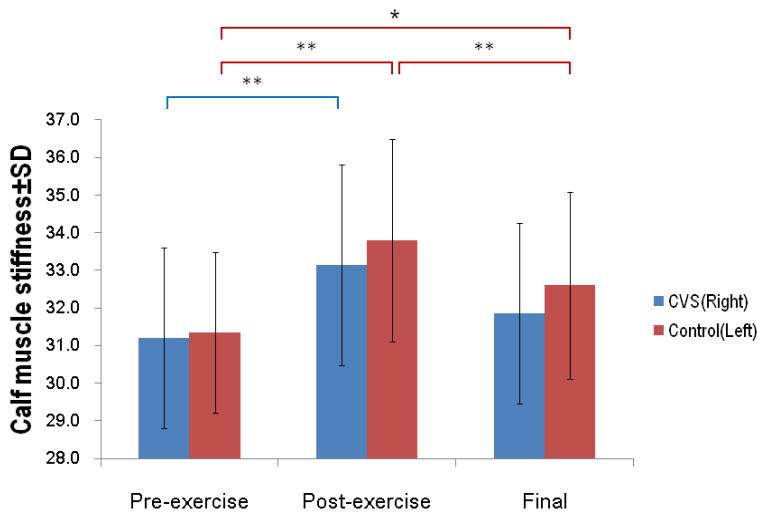

Figure 2. Multiple comparisons among pre-exercise, postexercise and final measurements of right and left calf muscle stiffness $(*: \mathrm{p}<0.05, * *: \mathrm{p}<0.01)$.

stored post-exercise calf circumference to baseline level. Exercise caused a significant increase in calf circumference and muscle stiffness (calf circumference: right and left: $\mathrm{p}<0.001$, muscle stiffness: left: $\mathrm{p}<0.001$, right: $\mathrm{p}$ $=0.007)$. The main cause of increased calf circumference was augmented blood flow and retention of tissue fluids in the working muscles. The major factors that led to greater stiffness included shortening of muscle tissue, changes in tensile strength of the muscle fibers, and accumulation of blood and body fluids.

The final circumference of the CVS-applied (right) leg reduced significantly compared with post-exercise circumference ( $\mathrm{p}<0.001)$, to a near baseline level. With respect to final stiffness, a trend towards improvement to a near baseline level was observed when compared with post-exercise stiffness, yet the difference was not significant $(p=0.080)$. We surmised that CVS application promoted the reduction in calf circumference and muscle stiffness by relaxing shortened muscle tissues and im- proving circulation.

In contrast, the circumference of the control leg decreased to a certain level after the exercise; however, it remained significantly greater than baseline even after 15 minutes of stopping the exercise $(p=0.025)$. A significant improvement in stiffness was observed after immobilization ( $\mathrm{p}=0.035)$; nevertheless the level of stiffness remained significantly greater than baseline 15 minutes after the exercise $(p=0.027)$. These results suggest that natural recovery via immobilization did not recover stiffness to the baseline level.

Our results suggest that recovery care with CVS markedly reduces calf circumference, but the correlation between reduced calf size and amelioration of muscle pain and fatigue after physical activity remain unexplained. In this study, we evaluated the acute effects of CVS in recovery, but further studies are warranted to investigate the effect of recovery care in muscle pain and fatigue one day after the workout. Several divergent views abound regarding the efficacy of stretching and massage for post-workout recovery. While stretching has been promoted for years as an essential part of a fitness program, Herbet et al. reported that post-exercise stretching failed to prevent muscle pain [7,8]. Moreover, Thacker et al. refuted the benefits of stretching before and after a workout in preventing sports injuries [9]. Furthermore, Johnhagen et al. reported that centripetal sports massage after physical activity did not lead to functional recovery of the muscles [10].

In contrast, Mastubashi et al. reported that stretching was effective in reducing muscle stiffness after a workout [11]. However, Matsubashi also cautioned that selfstretch had no effect on muscle stiffness and that noticeable improvement required proficiency in techniques [11]. Our particular method of recovery care did not yield a significant improvement in muscle stiffness; however, a more accurate evaluation of stiffness may be obtained by revising the methodology and conducting an assessment after repeated intervention.

Although our results confirm the general efficacy of recovery care with CVS, the effect of glutamic and citric acid-containing CVS itself requires further evaluation. For this purpose, a comparative analysis of recovery care with vaseline is necessary in the future. A more stringent evaluation under controlled environment is required to eliminate the blind faith in the effectiveness of postexercise stretching and massage held by some of the health care professionals, athletic trainers and therapists.

\section{CONCLUSION}

We conducted a post-workout recovery care with CVS to investigate its effect in preventing sports injury and hastening recovery, and measured calf circumference 
and muscle stiffness before and after the intervention. Our results showed that recovery care with CVS induced a significant reduction in calf circumference to pre-exercise level.

\section{REFERENCES}

[1] SSF Sasakawa Sports Foundation (2009) Sports Life Data 2008, SSF Sasakawa Sports Foundation.

[2] Ministry of Health, Labor, and Welfare (2008) National Health and Nutrition Survey. http://www.mhlw.go.jp/houdou/2009/11/h1109-1.html

[3] Yamamoto, T. (1990) Sports and Stretching. Physical Therapy, 7, 351-361.

[4] Japan Sports Association, 2009. http://www.japan-sports.or.jp/coach/data/data.html

[5] National Athletic Trainers' Association, 2010. http://www.nata.org

[6] Zhang, H., Matsuda, H., Yamashita, C., Nakamura, S. and Yoshikawa, M. (2009) Hydrangeic acid from the processed leaves of Hydrangea macrophylla var. thun- bergii as a new type of anti-diabetic compound. European Journal of Pharmacology, 606, 255-261.

[7] Herbert, R.D. and Gabriel, M. (2002) Effects of stretching before and after exercising on muscle soreness and risk of injury: systematic review. British Medical Journal, 325, 468.

[8] Herbert, R.D. and de Noronha, M. (2007) Stretching to prevent or reduce muscle soreness after exercise. Cochrane Database Systematic Reviews, 4. Art. No.: CD004577.

[9] Thacker, S.B., Gilchrist, J., Stroup D.F. and Kimsey Jr, C. D. (2004) The impact of stretching on sports injury risk: A systematic review of the literature. Medicine \& Science in Sports \& Exercise, 36, 371-378.

[10] Jonhagen, S., Ackermann, P., Eriksson, T., Saartok, T. and Renstrom, P.A. (2004) Sports massage after eccentric exercise. The American Journal of Sports Medicine, 32, 1499-1503.

[11] Matsuhashi, A., Sato, T., Asahina, S. and Kume, T. (2002) The effect of stretching on hardnessof the soleus muscle after tiptoe standing. The Showa University Journal of Medical Sciences, 62, 401-406. 